\title{
Inibição da Proliferação Neointimal Após o Implante de Stents Eluidores de Biolimus A9: Análise Volumétrica Tardia com Ultra-Som Intracoronário
}

\author{
Eduardo Missel1, Pedro Beraldo¹, Alexandre Abizaid', Luiz Alberto P. Mattos¹, Fausto Feres ${ }^{1}$, \\ Rodolfo Staico ${ }^{1}$, José de Ribamar Costa ${ }^{1}$, Felipe Cesar ${ }^{1}$, Marinella Centemero ${ }^{1}$, Áurea Chaves ${ }^{1}$, \\ Andrea Abizaid'1, Amanda Sousa', Eberhard Grube², J. Eduardo Sousa ${ }^{1}$
}

\section{RESUMO}

Introdução: O estudo STEALTH I demonstrou a eficácia e segurança dos stents eluidores de Biolimus A9 (BA9) em reduzir a perda-tardia angiográfica e apresentar uma baixa incidência de eventos cardíacos maiores, entretanto os achados de ultra-som intracoronário (USIC) ainda não foram descritos. O objetivo deste estudo é descrever os achados de análise volumétrica por USIC nos pacientes recrutados em nossa instituição. Métodos e Resultados: Quarenta e cinco pacientes apresentando lesão coronária de novo, única, foram randomizados 2:1 para receber stents eluidores de BA9 $(n=30)$ ou stents controle $(n=15)$. A média de idade foi de 58 anos, com 16\% diabéticos e $62 \%$ do sexo masculino. As características clínicas e angiográficas foram similares entre os grupos. Aos 6 meses de seguimento não houve diferença significativa entre os grupos quanto a desfechos clínicos. O índice de hiperplasia intimal intra-stent foi inferior no grupo dos stents eluidores de BA9 em comparação ao grupo controle $(0.19+/-0.08$ vs. $2.71+/-0.50, \mathrm{P}<0.0001)$, assim como o percentual de obstrução intra-stent $(2.2+/-0.80 \%$ vs. $19.9+/-4.67 \%$, $\mathrm{P}<0.0001)$. Não foi observado nenhum caso de trombose do stent, formação aneurismática ou aposição incompleta das hastes dos stents aos 6 meses de seguimento. Conclusões: Os stents eluidores de BA9 são altamente eficazes em inibir a proliferação neointimal intra-stent quando comparados aos stents controle, demonstrando um percentual mínimo de obstrução intra-stent aos 6 meses de seguimento.

DESCRITORES: Ultra-som. Contenedores. Reestenose coronária. Materiais revestidos biocompatíveis. Sirolimo, análogos \& derivados.

\section{SUMMARY}

\author{
Supression of Intimal Proliferation after \\ Implantation of Biolimus A9-Eluting Stents: \\ 6-Month Single-Center Intravascular \\ Ultrasound Follow-Up
}

Background: Biolimus A9 (BA9) is a novel sirolimus analog with similar antiproliferative properties. BA9-eluting stents reduce angiographic late-loss compared to bare metal stents (BMS), with a low incidence of major adverse cardiac events (MACE), as reported in the randomized, controlled STEALTH I trial. We describe the intravascular ultrasound (IVUS) volumetric findings of patients enrolled at our institution. Methods and Results: Forty-five patients presenting a single, de novo coronary lesion (2.5-4.0 mm vessels; lesion length $<24 \mathrm{~mm}$ ) were randomly assigned in a 2:1 basis to receive either BA9-eluting $(n=30)$ or bare metal stents $(n=15)$. Mean age was 58 years, with $16 \%$ diabetics and $62 \%$ men. Baseline clinical and angiographic characteristics were similar between groups. At 6 month follow-up, there was no statistical difference in clinical outcomes between groups. In-stent intimal hyperplasia index measured by IVUS was $0.19+/-0.08 \mathrm{~mm}^{3} /$ $\mathrm{mm}^{3}$ for the BA9 group vs. $2.71+/-0.50 \mathrm{~mm}^{3} / \mathrm{mm}^{3}$ for BMS controls. The percentage of stent obstruction was also significantly lower for the BA9 group when compared to BMS $(2.2+/-0.80 \%$ vs. $19.9+/-4.67 \%, \mathrm{P}<0.0001)$. No stent thrombosis, aneurysm formation or incomplete stent apposition was observed in any group. Conclusions: Using IVUS volumetric analysis, we showed that BA9-eluting stents have a potent inhibitory effect on neointimal proliferation when compared to BMS at 6-month follow-up.

DESCRIPTORS: Ultrasonics. Stents. Coronary restenosis. Coated materials, biocompatible. Sirolimus, analogs \& derivatives.

\footnotetext{
1 Instituto Dante Pazzanese de Cardiologia - São Paulo - Brazil 2 HELIOS Heart Center - Siegburg - Alemanha.

Correspondência: Alexandre Abizaid. Instituto Dante Pazzanese de Cardiologia. Rua Dante Pazzanese, 500 - São Paulo - SP - Brasil CEP 04038-003 - Fone: 55-11-5085-4141 Fax: 55-11-5549-7807 E-mail: aabizaid@uol.com.br Patrocinador do estudo: Biosensors International - Singapura. Recebido em: 3/5/2007 • Aceito em: 23/6/2007
} 


\section{$\mathbf{0}$} s stents eluidores de sirolimus e paclitaxel, que representam a primeira geração de stents farmacológicos disponíveis no mercado, são altamente eficazes no tratamento da doença arterial coronária, com uma sobrevida livre de eventos superior a 90\% dos pacientes tratados, mesmo em situações de alta complexidade clínica e angiográfica ${ }^{1-3}$. Entretanto, evidências recentes têm gerado dúvidas quanto à segurança destes dispositivos a longo prazo ${ }^{4}$, gerando um crescente interesse em novos programas de stents farmacológicos.

O biolimus A9 (Figura 1) é um fármaco análogo do sirolimus recentemente descoberto, com propriedades antiproliferativas semelhantes, promovendo o bloqueio da fase G1-S do ciclo celular. Assim como o sirolimus, inibe a proliferação neointimal in vitro às custas de um efeito contra-regulatório e citostático sobre as células musculares lisas $(\mathrm{CML})$, impedindo a proliferação e migração das mesmas da camada média para a íntima, exercendo assim seu efeito anti-reestenótico ${ }^{5}$. Desta forma, tanto o biolimus A9 quanto o sirolimus apresentam propriedades químicas e mecanismo de ação distintos ao paclitaxel, que por sua vez, exerce efeito tóxico no sistema microtubular, impedindo a mitose das CML e desencadeando mecanismos pró-apoptóticos ${ }^{6}$.

Os resultados da primeira experiência com os stents eluidores de Biolimus A9 em humanos foram reportados recentemente, com resultados angiográficos de 6 meses altamente superiores ao grupo controle e baixa incidência global de eventos clínicos maiores, porém os dados de ultra-sonografia intracoronária ainda não foram reporta$\operatorname{dos}^{7}$. O objetivo deste estudo é avaliar o grau de proliferação neointimal intra-stent destas novas endopróteses, no seguimento de 6 meses dos pacientes recrutados em nossa instituição, utilizando para tal a análise volumétrica pela ultra-sonografia intracoronária.

\section{MÉTODO}

\section{Protocolo do estudo e definições}

No período de novembro de 2003 a março de 2004, 49 pacientes foram recrutados em nosso centro

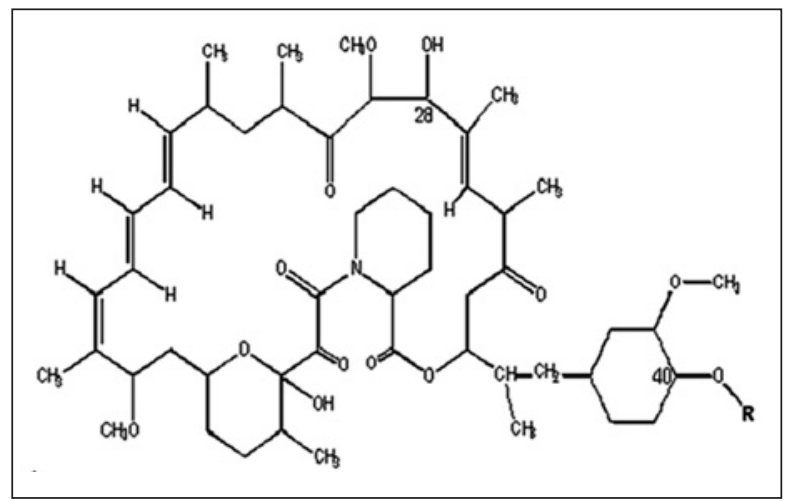

Figura 1 - Estrutura molecular do Biolimus A9. e randomizados 2:1 para receber o stent eluidor de BA9 BioMatrix ${ }^{\mathrm{TM}}$ (Biosensors International, Singapura) $(n=32)$ ou o stent convencional S-stent ${ }^{\mathrm{TM}}$ (Biosensors International, Singapura) $(n=17)$. Três pacientes não realizaram o reestudo ultra-sonográfico por dificuldades técnicas inerentes ao procedimento (tortuosidades excessivas do vaso associadas à incapacidade de cruzar a borda proximal da endoprótese, sem evidência de hiperplasia acentuada à angiografia nos três casos), sendo excluídos desta análise independente do tipo de stent implantado. A amostra analisada, portanto, foi composta por 30 pacientes no grupo do Biolimus A9 e 15 pacientes no grupo controle.

Os critérios de inclusão foram lesão coronária de novo, única, com diâmetro de estenose de 50 a 99\%, diâmetro de referência angiográfico de 2,75 a 4,0 mm e extensão da lesão inferior a $24 \mathrm{~mm}$. A pré-dilatação da lesão foi mandatória. Pacientes com idade inferior a 18 anos, apresentando lesão significante em tronco de coronária esquerda ( $>50 \%)$, oclusão crônica total, fluxo TIMI inferior a 2, bifurcações envolvendo ramo secundário significante (diâmetro $>2 \mathrm{~mm}$ ), lesões ostiais, choque cardiogênico, fração de ejeção do ventrículo esquerdo inferior a 30\%, infarto agudo do miocárdio (IAM) transmural há menos de uma semana ou necessitando implante de múltiplos stents foram excluídos do estudo. Todos os pacientes assinaram o documento de consentimento informado, e o protocolo do estudo foi aprovado pela comissão de ética da instituição.

IAM foi definido como novas ondas Q patológicas em duas ou mais derivações contínuas ou elevação da creatino-quinase (CK) ou sua isoenzima MB (CK-MB)

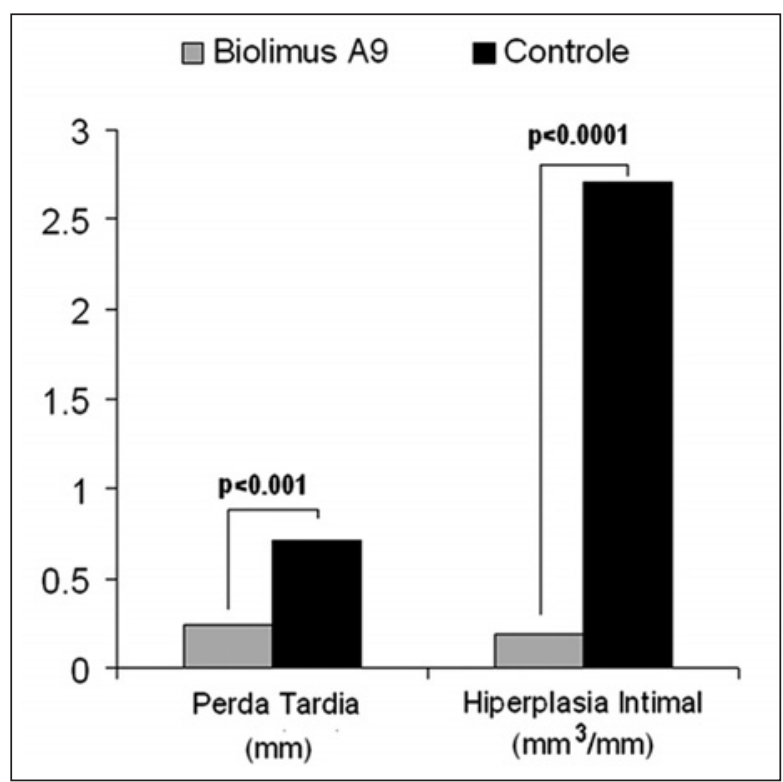

Figura 2 - Resultados concordantes entre angiografia quantitativa (perda tardia) e ultra-sonografia intracoronária (índex de hiperplasia intimal) quanto ao impacto do Biolimus A9 na inibição da proliferação neointimal. 
$>3$ vezes o valor superior da normalidade durante a hospitalização, ou $>2$ vezes o limite superior da normalidade após a mesma. CK e CK-MB foram dosadas antes do procedimento, 8-12 horas e 18-24 horas após, e então diariamente até a alta hospitalar.

\section{Farmacoterapia adjuvante}

Todos os pacientes receberam farmacoterapia adjuvante, sendo pré-tratados com aspirina em caso de não fazerem uso prévio (325 mg VO 24h antes do procedimento) e tienopiridínicos em dose de ataque (ticlopidina 500 mg VO ou clopidogrel 300 mg VO). Durante o procedimento, os pacientes receberam heparina não-fracionada intravenosa $(100 \mathrm{UI} / \mathrm{kg})$, com o objetivo de manter o tempo de coagulação ativado entre 250 e 350 segundos. Todos os pacientes receberam clopidogrel ou ticlopidina por, ao menos, seis meses após o implante de stents, e aspirina continuamente. Os inibidores de glicoproteína foram utilizados a critério dos operadores.

\section{Aquisição das imagens}

A angiografia pós-procedimento foi realizada em, ao menos, duas projeções ortogonais. As mesmas projeções ortogonais foram repetidas aos seis meses de seguimento. Realizamos, então, a aquisição de imagens dos stents implantados utilizando transdutor de elemento único, rotacional, com freqüência de $40 \mathrm{Mhz}$ (ClearView, Boston Scientific Corp, Nattick, EUA), sendo realizado recuo motorizado a uma velocidade $0,5 \mathrm{~mm} /$ seg. As imagens adquiridas foram, então, armazenadas em fitas s-VHS para posterior reconstrução tridimensional.

\section{Angiografia Coronária Quantitativa}

Dois investigadores independentes, sem conhecimento do tipo de stent empregado em cada grupo, realizaram análise coronária quantitativa utilizando um sistema de detecção automática de bordas (MEDIS Medical Imaging System, Leiden, Holanda). A ponta do cateter repleta de contraste foi utilizada para calibração. O diâmetro luminal mínimo pré e pós-procedimento, diâmetro de referência e diâmetro do balão em insuflação máxima foram mensurados. O ganho luminal agudo (DLM pós-procedimento - DLM pré-procedimento) e a perda angiográfica tardia (DLM intra-stent no reestudo - DLM intra-stent pós-procedimento) foram então determinados.

\section{Análise volumétrica por ultra-sonografia intracoronária}

A reconstrução tridimensional das imagens de ultrasonografia intracoronária foi realizada com auxílio do programa EchoPlaque 2 (Indec Systems Inc., Mountain View, EUA), por dois investigadores independentes cegos para o tratamento instituído. Os segmentos referentes aos stents foram medidos a cada milímetro por planimetria computadorizada. As áreas referentes ao stent, lúmen, e hiperplasia neointimal (área do stent - área do lúmen) foram medidas no segmento analisado, e os volumes foram calculados pela regra de Simpson ${ }^{8}$. O porcentual de obstrução volumétrica intra-stent (\%OV) foi calculado como a relação entre o volume de hiperplasia e o volume do stent X 100, e o índice de hiperplasia intimal (IHI) como a relação entre o volume absoluto de hiperplasia e a extensão do stent ${ }^{9}$. Aposição incompleta do stent foi definida como separação de uma ou mais hastes do stent da parede do vaso com presença de fluxo entre o stent e o vaso.

\section{Análise Estatística}

A análise estatística foi realizada com o programa StatView 5.0.1 (SAS Institute, Cary, EUA). As variáveis numéricas foram expressas como média \pm desvio-padrão (DP) e as categóricas como números (n) e porcentagens $(\%)$. O teste do qui-quadrado e o teste exato de Fisher foram usados para variáveis categóricas; o teste $\mathrm{t}$ de Student bicaudal foi utilizado nas comparações de variáveis contínuas. Valores de probabilidade $<0,05$ foram considerados estatisticamente significativos.

\section{RESULTADOS}

As características clínicas e angiográficas dos pacientes incluídos no estudo foram semelhantes entre os grupos (Tabela 1). Em ambos os grupos, não houve incidência de morte, acidente vascular cerebral ou sangramentos maiores. Dois $(6,6 \%)$ pacientes no grupo do Biolimus A9 apresentaram eventos cardíacos maiores: um (3,3\%) paciente apresentou IAM no seguimento de seis meses (não relacionado ao vaso-alvo), e um (3,3\%) necessitou de revascularização da lesão-alvo (Tabela 2).

\section{Angiografia Coronária Quantitativa}

A análise angiográfica quantitativa foi realizada em todos os pacientes (100\%). O diâmetro de referência do vaso tratado $(2,95 \pm 0,40$ vs. $2,97 \pm 0,43, p=0,87)$ e a extensão da lesão-alvo $(14,5 \pm 4,22$ vs. 12,75 $\pm 3,82$, $\mathrm{p}=0,18)$ foram semelhantes entre os grupos Biolimus A9 e o grupo controle. O ganho agudo foi de 2,14 $\pm 0,35$ para o grupo Biolimus A9 vs. 2,15 $\pm 0,32$ para o grupo controle $(p=0,92)$.

A perda tardia (PT) angiográfica intra-stent, aos seis meses de seguimento, foi significativamente inferior para o grupo Biolimus A9 vs. grupo controle $(0,24 \pm 0,39$ vs. $0,71 \pm 0,47 \mathrm{~mm}, \mathrm{p}<0,001)$. A reestenose binária ocorreu em apenas uma paciente no grupo do Biolimus A9, e não foi observada em nenhum paciente pertencente ao grupo controle $(p=0,51)$.

\section{Análise volumétrica pela ultra-sonografia intracoronária}

A análise volumétrica intra-stent pela ultra-sonografia intracoronária aos seis meses foi realizada em todos os pacientes incluídos nesta análise, e está descrita na Tabela 3. Aos seis meses de seguimento, não houve diferença significativa quanto ao volume do stent ou volume do lúmen entre os grupos. A extensão dos 
Missel E, et al. Inibição da Proliferação Neointimal Após o Implante de Stents Eluidores de Biolimus A9: Análise Volumétrica Tardia com Ultra-Som Intracoronário. Rev Bras Cardiol Invas 2007; 15(3): 234-239.

stents analisados também foi semelhante. Os pacientes que receberam o implante de stents eluidores de BA9 apresentaram valores significativamente inferiores para o porcentual de obstrução volumétrica intra-stent. $\mathrm{O}$ volume de hiperplasia por milímetro de stent $(\% \mathrm{IHI})$ foi reduzido em $86 \%$ no grupo do biolimus A9 em comparação ao grupo controle (Figura 2). Na análise qualitativa de seis meses, não foi observado nenhum caso de formação aneurismática ou má aposição das hastes dos stents em ambos os grupos.

\section{DISCUSSÃO}

Os principais achados deste estudo são: (1) o stent eluidor de Biolimus A9 é altamente superior aos stents controle em inibir a proliferação neointimal e (2) os achados de ultra-sonografia intracoronária confirmam os resultados angiográficos previamente descritos.

O Biolimus A9 é um dos fármacos descobertos recentemente pertencente à mesma classe farmacológica

TABELA 1

Características clínicas e angiográficas

\begin{tabular}{lcc}
\hline & $\begin{array}{c}\text { Biolimus A9 } \\
\mathbf{n = 3 0}\end{array}$ & $\begin{array}{c}\text { Controle } \\
\mathbf{n = 1 5}\end{array}$ \\
\hline Idade & $58,3 \pm 9,1$ & $57,2 \pm 7,5$ \\
Homens & $15(50)$ & $11(73)$ \\
Diabetes & $6(20)$ & $2(13)$ \\
Hipertensão & $24(80)$ & $14(93)$ \\
Tabagismo & $16(53)$ & $12(80)$ \\
Dislipidemia & $19(63)$ & $5(33)$ \\
Hx Familiar & $13(43)$ & $5(33)$ \\
IAM prévio & $15(50)$ & $9(60)$ \\
ATC prévia & $2(7)$ & $0(0)$ \\
CRM prévia & $4(13)$ & $1(7)$ \\
Angina Instável* & $18(6)$ & $11(73)$ \\
Angina Estável* & $12(40)$ & $4(27)$ \\
I & $1(3)$ & $1(7)$ \\
II & $9(30)$ & $3(20)$ \\
III & $1(3)$ & $1(7)$ \\
IV & $0(0)$ & $0(0)$ \\
Lesões* & $18(60)$ & $10(67)$ \\
A/B1 & $12(40)$ & $5(33)$ \\
B2/C & $10(33)$ & $5(33)$ \\
Vaso Tratado & $8(27)$ & $5(33)$ \\
DA & $12(40)$ & $53)$ \\
CX & & \\
CD & & \\
& &
\end{tabular}

*Classificação angiográfica e definições de acordo com American College of Cardiology/American Heart Association. Todos $\mathrm{p}=\mathrm{NS}$. IAM= Infarto agudo do miocárdio, ATC= Angioplastia transluminal coronária, $\mathrm{CRM}=$ Cirurgia de revascularização do miocárdio. do Sirolimus, caracterizada por propriedades macrolídeas e antifúngicas, exercendo um potente efeito citostático nas células musculares lisas ${ }^{4}$. O estudo STEALTH I foi desenhado para avaliar a segurança e eficácia dos stents eluidores de Biolimus A9 no tratamento de 120 lesões coronarianas de novo. Os resultados clínico-angiográficos foram recentemente reportados por Costa et al. ${ }^{7}$, demonstrando superioridade na perda tardia angiográfica em relação aos stents convencionais $(0,26 \pm 0,43$ vs $0,74 \pm 0,45 \mathrm{~mm}, \mathrm{p}<0,001)$, e uma baixa incidência de eventos cardíacos maiores aos seis meses de seguimento. Os resultados de análise volumétrica em nosso estudo são concordantes com estes achados, com resultados de perda tardia angiográfica superponíveis e demonstrando proliferação neointimal intra-stent, à ultra-sonografia intracoronária, mínima, no grupo do Biolimus A9. Adicionalmente, os valores encontrados para porcentual de hiperplasia intimal intra-stent (também denominado porcentual de obstrução intra-stent) foram comparavelmente baixos em relação aos resultados de ultra-sonografia tridimensional reportados previamente para os stents eluidores de sirolimus. Serruys et al. ${ }^{10}$ reportaram um volume de obstrução intra-stent de $1,0 \pm 3,0 \%$ para o grupo dos stents eluidores de sirolimus vs. $37,0 \pm 28,0 \%$ para o grupo controle $(p<0,001)$, enquanto em nosso estudo o valor encontrado foi de $2,2 \pm 0,8 \%$ para os stents eluidores de Biolimus A9 vs. 19,9 $\pm 4,67$ para o grupo controle $(p<0,0001)$. Portanto, analisando os dados disponíveis para ambos os estudos, os stents eluidores de sirolimus e biolimus A9 apresentam uma redução superior a 90\% na proliferação neointimal, aos seis meses de seguimento, quando comparados aos stents convencionais.

Apesar da superioridade angiográfica e ultra-sonográfica do BA9 em relação ao grupo controle, em nossa análise, não houve diferença em relação à reestenose binária entre os grupos, havendo inclusive uma curiosa "inversão" nos resultados clínicos. Entretanto,

TABELA 2

Seguimento clínico de seis meses

\begin{tabular}{lcc}
\hline & $\begin{array}{c}\text { Biolimus A9 } \\
\mathbf{n = 3 0}\end{array}$ & $\begin{array}{c}\text { Controle } \\
\mathbf{n = 1 5}\end{array}$ \\
\hline Eventos cardíacos maiores \# (\%) & $2(6,7)$ & 0 \\
Óbito \# (\%) & 0 & 0 \\
IAM \# (\%) & $1(3,3)^{*}$ & 0 \\
AVC \# (\%) & 0 & 0 \\
RLA-ATC \# (\%) & $1(3,3)$ & 0 \\
RLA-CRM \# (\%) & 0 & 0
\end{tabular}

*Não relacionado ao vaso-alvo. Todos $p=N S$. IAM= Infarto agudo do miocárdio, $\mathrm{AVC}=$ Acidente vascular cerebral, $\mathrm{RLA}=$ Revascularização da lesão-alvo, ATC= Angioplastia transluminal coronária, CRM= Cirurgia de revascularização do miocárdio. 
Missel E, et al. Inibição da Proliferação Neointimal Após o Implante de Stents Eluidores de Biolimus A9: Análise Volumétrica Tardia com Ultra-Som Intracoronário. Rev Bras Cardiol Invas 2007; 15(3): 234-239.

TABELA 3

Análise volumétrica intra-stent pela ultra-sonografia intracoronária (USIC) aos seis meses

\begin{tabular}{lccr}
\hline & $\begin{array}{c}\text { Biolimus A9 } \\
\mathbf{n = 3 0}\end{array}$ & $\begin{array}{c}\text { Controle } \\
\mathbf{n = 1 5}\end{array}$ & $\mathbf{p}$ \\
\hline Extensão do stent $(\mathrm{mm})$ & $19,26 \pm 6,04$ & $18,13 \pm 5,42$ & 0,54 \\
Volume do stent $\left(\mathrm{mm}^{3}\right)$ & $162,55 \pm 80,2$ & $167,81 \pm 65,2$ & 0,83 \\
Volume do lúmen $\left(\mathrm{mm}^{3}\right)$ & $159,35 \pm 58,2$ & $139,23 \pm 54,1$ & 0,27 \\
Índex de hiperplasia intimal $\left(\mathrm{mm}^{3} / \mathrm{mm}\right)$ & $0,19 \pm 0,08$ & $2,71 \pm 0,50$ & $<0,0001$ \\
Obstrução volumétrica intra-stent $(\%)$ & $2,2 \pm 0,80$ & $19,9 \pm 4,67$ & $<0,0001$ \\
\hline
\end{tabular}

antes de qualquer conclusão a esse respeito devem ser considerados os seguintes fatos: (1) um paciente no grupo do Biolimus A9 apresentou IAM não relacionado ao vaso-alvo, (2) o número de pacientes no grupo controle é muito pequeno (15 pacientes), favorecendo resultados ao acaso, como pode ser observado pelo valor de probabilidade não significante entre os grupos quanto a desfechos clínicos. Outro aspecto que merece maior esclarecimento é o de que, nos três pacientes excluídos do estudo, o motivo foi a anatomia coronariana local desfavorável para progressão do cateter de ultrasonografia intracoronária, e não hiperplasia intra-stent acentuada. Adicionalmente, os investigadores desconheciam o grupo a qual os pacientes excluídos pertenciam, eliminando-se, assim, um potencial vício implícito.

As diferenças entre a molécula do Sirolimus e Biolimus A9 são primariamente estruturais e conferem propriedades mais lipofílicas ao Biolimus A9, sendo esperada uma eluição mais rápida. A plataforma do stent eluidor de sirolimus possui um desenho tubular em células fechadas, enquanto o S-stent (plataforma do BA9) apresenta desenho tubular em quadratura, com o objetivo de aumentar a flexibilidade da endoprótese. Talvez a diferença mais marcante seja em relação ao polímero: o stent com sirolimus emprega um polímero durável, que permanece aderido ao stent após a eluição do fármaco em 28 dias, já o stent eluidor de BA9 emprega um polímero bioabsorvível o ácido polilático (PLA) - que desaparece totalmente após o período de eluição, sendo liberado juntamente com o fármaco na parede do vaso.

Evidências histopatológicas recentes têm atribuído aos polímeros duráveis reações de hipersensibilidade local, com infiltração eosinofílica, resultando em um maior potencial trombótico, além de um possível papel na gênese da má aposição tardia dos stents ${ }^{11}$. Este último fenômeno, definido como a separação de uma ou mais hastes do stent da parede do vaso observada somente no seguimento tardio ${ }^{12}$, foi observado no seguimento ultra-sonográfico da maioria dos grandes estudos randomizados com o stent eluidor de sirolimus ${ }^{13-15}$. Os investigadores dos estudos FUTURE I e II ${ }^{16,17}$, que avaliaram a segurança e eficácia dos stents eluidores de everolimus empregando o PLA como polímero bioab- sorvível, não observaram este fenômeno no seguimento seriado de seis e 12 meses, contribuindo para a hipótese de que os polímeros bioabsorvíveis não apresentam efeitos deletérios na superfície interna do segmento arterial tratado. Apesar de somente uma análise seriada de ultra-sonografia intracoronária a longo prazo, com maior amostragem, ser capaz de avaliar adequadamente a presença ou não do fenômeno de má aposição tardia, o fato de não termos nenhum caso de aposição incompleta no seguimento de seis meses do nosso estudo é consistente com este conceito.

\section{Limitações do estudo}

A amostra limitada deste estudo impede uma comparação adequada dos stents eluidores de BA9 com o grupo controle em relação a desfechos clínicos ou reestenose binária. O período de seguimento restrito a seis meses ainda não nos permite acessar a eficácia e segurança tardia desta nova geração de stents.

\section{CONCLUSÕES}

Os stents eluidores de Biolimus A9 exercem um potente efeito inibitório na proliferação neointimal aos 6 meses de seguimento, com um porcentual de obstrução volumétrica intra-stent significativamente inferior aos stents convencionais.

\section{REFERÊNCIAS BIBLIOGRÁFICAS}

1. Stone GW, Moses JW, Ellis SG, Schofer J, Dawkins KD, Morice MC, et al. Safety and efficacy of sirolimus- and paclitaxel-eluting coronary stents. N Engl J Med. 2007;356(10): 998-1008.

2. Morice MC, Colombo A, Meier B, Serruys P, Tamburino C, Guagliumi G, et al. Sirolimus- vs paclitaxel-eluting stents in de novo coronary artery lesions: the REALITY trial: a randomized controlled trial. REALITY Trial Investigators. JAMA. 2006;295(8):895-904.

3. Sousa JE, Missel E, Abizaid A, Sousa A. Are paclitaxeleluting stents safe and effective in patients with acute coronary syndromes? Nat Clin Pract Cardiovasc Med. 2005;2 (11):566-7.

4. lakovou I, Schmidt T, Bonizzoni E, Ge L, Sangiorgi GM, Stankovic G, et al. Incidence, predictors, and outcome of thrombosis after successful implantation of drug-eluting stents. JAMA. 2005;293(17):2126-30.

5. Kar S, Aragon J, Paresky A, Tio F, Shulze J, Betts R, et al. 
Missel E, et al. Inibição da Proliferação Neointimal Após o Implante de Stents Eluidores de Biolimus A9: Análise Volumétrica Tardia com Ultra-Som Intracoronário. Rev Bras Cardiol Invas 2007; 15(3): 234-239.

1139-52 Biolimus A9: a new generation rapamycin analogue inhibits intimal hyperplasia in a porcine model. J Am Coll Cardiol. 2004;43(supll):A84.

6. Wessely R, Schömig A, Kastrati A. Sirolimus and paclitaxel on polymer-based drug-eluting stents: similar but different. J Am Coll Cardiol. 2006;47(4):708-14.

7. Costa RA, Lansky AJ, Abizaid A, Müeller R, Tsuchiya Y, Mori K, et al. Angiographic results of the first human experience with the Biolimus A9 drug-eluting stent for de novo coronary lesions. Am J Cardiol. 2006;98(4):443-6.

8. Mintz GS, Nissen SE, Anderson WD, Bailey SR, Erbel R, Fitzgerald PJ, et al. American College of Cardiology Clinical Expert Consensus Document on Standards for Acquisition, Measurement and Reporting of Intravascular Ultrasound Studies (IVUS). A report of the American College of Cardiology Task Force on Clinical Expert Consensus Documents. J Am Coll Cardiol. 2001;37(5):1478-92.

9. Mehran R, Mintz GS, Hong MK, Tio FO, Bramwell O, Brahimi $A$, et al. Validation of the in vivo intravascular ultrasound measurement of in-stent neointimal hyperplasia volumes. J Am Coll Cardiol. 1998;32(3):794-9.

10. Serruys PW, Degertekin M, Tanabe K, Abizaid A, Sousa JE, Colombo A, et al. Intravascular ultrasound findings in the multicenter, randomized, double-blind RAVEL (RAndomized study with the sirolimus-eluting VElocity balloon-expandable stent in the treatment of patients with de novo native coronary artery Lesions) trial. RAVEL Study Group. Circulation. 2002;106(7):798-803.

11. Joner M, Finn AV, Farb A, Mont EK, Kolodgie FD, Ladich $\mathrm{E}$, et al. Pathology of drug-eluting stents in humans: dela- yed healing and late thrombotic risk. J Am Coll Cardiol. 2006;48(1):193-202.

12. Serruys PW, Degertekin M, Tanabe K, Abizaid A, Sousa JE, Colombo A, et al. Intravascular ultrasound findings in the multicenter, randomized, double-blind RAVEL (Randomized study with the sirolimus-eluting Velocity balloon-expandable stent in the treatment of patients with de novo native coronary artery Lesions) trial. RAVEL Study Group. Circulation. 2002;106(7):798-803.

13. Degertekin M, Serruys PW, Tanabe $K$, Lee $C H$, Sousa JE, Colombo A, et al. Long-term follow-up of incomplete stent apposition in patients who received sirolimus-eluting stent for de novo coronary lesions: an intravascular ultrasound analysis. Circulation. 2003;108(22):2747-50.

14. Virmani R, Guagliumi G, Farb A, Musumeci G, Grieco N, Motta $\mathrm{T}$, et al. Localized hypersensitivity and late coronary thrombosis secondary to a sirolimus-eluting stent: should we be cautious? Circulation. 2004;109(6):701-5.

15. Ako J, Morino Y, Honda Y, Hassan A, Sonoda S, Yock PG, et al. Late incomplete stent apposition after sirolimus-eluting stent implantation: a serial intravascular ultrasound analysis. J Am Coll Cardiol. 2005;46(6):1002-5.

16. Grube E, Sonoda S, Ikeno F, Honda Y, Kar S, Chan C, et al. Six- and twelve-month rersults from first human experience using everolimus-eluting stents with bioabsorbable polymer. Circulation. 2004;109(18):2168-71.

17. Tsuchiya Y, Lansky AJ, Costa RA, Mehran R, Pietras C, Shimada $\mathrm{Y}$, et al. Effect of everolimus-eluting stents in different vessel sizes (from the pooled FUTURE I and II trials). Am J Cardiol. 2006;98(4):464-9. 